\title{
Approximate Solution of Dam-break Flow of Low Viscosity Bingham Fluid
}

\author{
How Tion PUAY* and Takashi HOSODA** \\ * Member Phd. Dept. of Urban Management Kyoto University (Kyodai Katsura, Nishikyo-ku, Kyoto 615-8540) \\ ** Member Prof. Dept. of Urban Management Kyoto University (Kyodai Katsura, Nishikyo-ku, Kyoto 615-8540)
}

In this study, we investigate the characteristics of dam-break flow of low viscosity Bingham fluid by deriving an approximate solution for the time development of the front position and depth at the origin of the flow. The asymptotic solutions representing the characteristic of Bingham fluid in the limit of low plastic viscosity are verified with a depth-averaged numerical model. Numerical simulations showed that with the decrease of plastic viscosity, the time development of the front position and depth at the origin approach to the theoretical asymptotic solution.

Key Words: Bingham fluid, slump flow, dam-breakflow

\section{Introduction}

The study of Bingham fluid flow characteristic is important due to its ubiquity in our life. In nature, mudflow which is often in the form of mixture of water with silt or volcanic ash (known as volcanic mudflow) is shown to behave like Bingham fluid ${ }^{1)}$. In view of the economy and life loss due to mudflow, the study of its flow behavior is indispensable. Fresh concrete is another example of fluid that flow behavior can be approximated by Bingham fluid constitutive law ${ }^{3}$. The knowledge of the relationship between the rheological properties and flow behavior of fresh concrete is important for the better handling of fresh concrete and to make sure that the formwork is properly filled. A slump flow test is a common method to investigate the rheological-flow relationship of fresh concrete, and can be treated as a kind of dam-break flow of Bingham fluid with plastic viscosity $\mu$ and yield stress $\tau_{y}{ }^{2), 3)}$.

Hosoda, et al. ${ }^{2)}$ pointed out that, in the case of low yield stress $\tau_{y}$, the characteristics of slump flow of Bingham fluid can be explained by the time development of the front position and the height at the origin of dam-break flow of finite volume viscous Newtonian fluid. These characteristics are denoted by the power law of time. It is also shown that with the decrease of plastic viscosity, the time development curve of the front approaches to one asymptotic curve. Fig. 1 shows the time development curve of the front of slump flow tests with almost constant yield stress conducted by Kokado, et al. ${ }^{3), 4}$. As pointed out by Hosoda, et $\mathrm{al}^{2}{ }^{2}$, it is worth to note that the time development curves lean towards an asymptotic curve, which is characterized by $1 / 1$

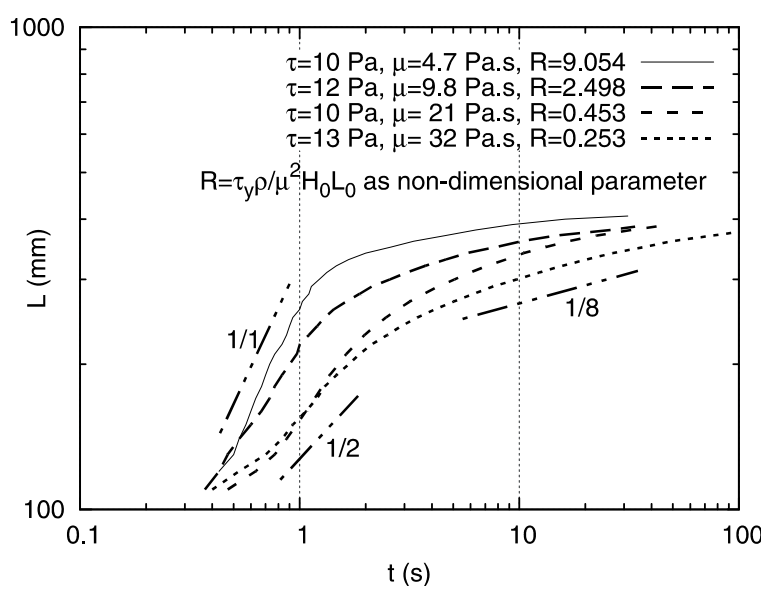

Fig.1 Time development of front position of slump flow test.

slope with the decrease of plastic viscosity. Following such observation, our motive in this study it to derive such asymptotic curve in the limit of low plastic viscosity, for a problem set in Cartesian coordinate system, which will be our first step towards obtaining a theoretical curve capable of describing the time development of the front of slump flow for a practical range of $\mu$ and $\tau_{y}$ values in the near future.

Based on shallow flow approximation, Huppert ${ }^{5)}$ and Liu, et al. ${ }^{6}$ used a one-dimensional model to study viscous Newtonian and Bingham fluid flow characteristics respectively. In this study, we also choose to use a one-dimensional model in view of its mathematical and numerical simplicity and our aim to derive a simple asymptotic solution. We first transform the basic governing equations into a moving coordinate system rescaled by the front position of the flow. By assuming the functional 


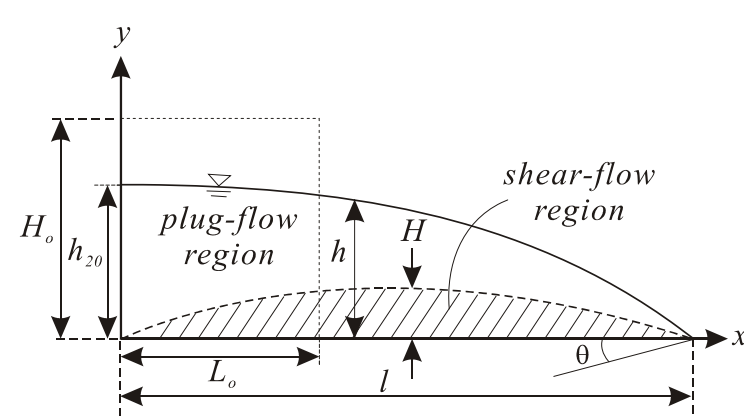

Fig.2 Proposed Bingham fluid model with plug-flow and shear-flow regions.

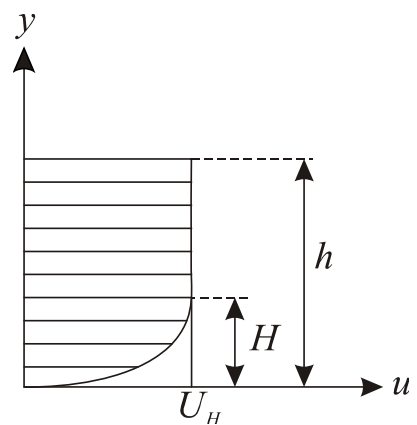

Fig.3 Velocity distribution of proposed Bingham model

form of the depth profile of the flow, the characteristic variables (front position of the flow and depth at the origin) are expressed in a set of ordinary differential equations (ODE). By solving the ODEs, the approximate asymptotic solutions of the time development of front position of flow in the limit of low plastic viscosity can be derived.

We use a numerical model which consists of a set of depth averaged flow equations with the resistance law based on the Bingham fluid's constitutive relation to verify the theoretical findings. Several simulation cases with varying plastic viscosity $\mu$, under constant yield stress $\tau_{y}$ showed the shifting of the time development curve of front-position towards the asymptotic solution. Numerical results also showed the influence of yield stress $\tau_{y}$ on the viscous phase of the flow. In this paper, since the density of fresh concrete $\rho$ and the slump initial height $H_{o}$ and initial length $L_{0}$ are constant throughout all the cases considered here, we choose to retain the use of $\mu$ and $\tau_{y}$ in this manuscript instead of a set of non-dimensional parameters such as $Y \equiv \tau_{y} /\left(\rho g H_{o}\right)$ and $M \equiv \mu /\left(\rho \sqrt{g H_{0}^{3}}\right)$.

\section{Proposed Bingham Fluid Model}

The constitutive law of Bingham fluid can be written as follows,

$$
\tau_{y}+\mu \frac{\partial u}{\partial y}=\left\{\begin{array}{lll}
\tau & \text { if } \tau>\tau_{y} \\
0 & \text { if } \tau \leq \tau_{y}
\end{array}\right.
$$

where $\tau$ is the shear stress acting on the fluid body. The yield stress $\tau_{y}$ is a threshold value where deformation occurs only if $\tau>\tau_{y}$. We use Jiang and LeBlond's ${ }^{7)}$ proposed Bingham model where the fluid is assumed to be comprised of two regions, namely the shear-flow region and the plug-flow region as shown in Fig. 2. The shear-flow region is the region where yielding occurs, and is defined within the range of $0 \leq y \leq H$. The plug-flow region is the region where shear stress acting on the fluid is less than the yield stress value, and is defined within the region of $\mathrm{H}<y \leq h$. Based on the Bingham constitutive relation, no yielding of fluid body occurs in the plug-flow region. From Fig. 2, by assuming steady uniform flow condition, we can write the following relation,

$$
\tau=\rho g \sin \theta(h-y)
$$

By using Bingham fluid constitutive relation in Eq. (1), we express the shear stress in Eq. (2) in terms of $\tau_{y}$ and $\partial u / \partial y$ as follows,

$$
\tau_{y}+\mu \frac{\partial u}{\partial y}=\rho g \sin \theta(h-y)
$$

Since in the region of plug-flow, rate of strain $\partial u / \partial y=0$ as shown in Fig. 3, we can write the following relation,

$$
y=H, \frac{\partial u}{\partial y}=0, \quad \tau_{y}=\rho g \sin \theta(h-H)
$$

We substitute Eq. (4) into Eq. (3) for $\tau_{y}$ and integrate Eq. (3) for the whole depth with boundary condition of $y=0, u=0$ to obtain the velocity profile of the flow as in Eq. (5),

$$
u=\frac{\rho g \sin \theta}{\mu}\left(H y-\frac{1}{2} y^{2}\right)
$$

The velocity profile in Eq. (5) is similiar to the velocity profile of steady uniform flow of Newtonian fluid in the absense of $\tau_{y}$, which also means $H=h$. By substituting Eq. (4) into Eq. (5), the velocity profile can be written as follows,

$$
u=\frac{\tau_{y}}{\mu(h-H)}\left(H y-\frac{1}{2} y^{2}\right)
$$

We can express the velocity of the plug-flow region $U_{H}$ by substituting $y=H$ in Eq. (6)

$$
U_{H}=\frac{\tau_{y} H^{2}}{2 \mu(h-H)}
$$

Therefore, the depth-averaged velocity of the flow, $U$ can be calculated as follows,

$$
\begin{aligned}
U & =\frac{(h-H) U_{H}+\int_{0}^{H} u d y}{h} \\
& =U_{H}\left(1-\frac{H}{3 h}\right)
\end{aligned}
$$

By using Eq. (6),

$$
\frac{\partial u}{\partial y}=\frac{\tau_{y}(H-y)}{\mu(h-H)}
$$


Consequently, we can therefore express $\tau_{b}$ in terms of yield stress $\tau_{y}$ and $H$ as follows,

$$
\tau_{b}=\tau_{y}+\left.\mu \frac{\partial u}{\partial y}\right|_{y=0}=\tau_{y} \frac{h}{(h-H)}
$$

\section{Theoretical Analysis}

\subsection{Characteristics of flow in the inertial phase}

1) Governing equations

The existence of inertial phase and its characteristic has been shown in several studies ${ }^{2), 8}$. In the following analysis, we study the characteristic of flow in the inertial phase which occurs at the initial stage of dam-break flow. We use the one-dimensional depth-averaged continuity and momentum equations,

Continuity equation,

$$
\frac{\partial h}{\partial t}+\frac{\partial U h}{\partial x}=0
$$

Momentum equation,

$$
\frac{\partial U h}{\partial t}+\frac{\partial U^{2} h}{\partial x}+g h \frac{\partial h}{\partial x}=-\tau_{b}
$$

2) Governing equations for low plastic viscosity Bingham fluid

In the case of low plastic viscosity, we assume that $H \rightarrow 0$. We rearrange Eq. (8) to obtain $U$ in the limit of $H \rightarrow 0$ as follows,

$$
\begin{gathered}
U=\frac{\tau_{y}}{2 \mu} \frac{H^{2}}{h(h-H)}\left(h-\frac{H}{3}\right) \\
H \rightarrow 0, \quad U=\frac{\tau_{y} H^{2}}{2 \mu h}
\end{gathered}
$$

It should be noted that Eq. (14) is derived based on the assumption that $H \ll h$. In addition, for the limit of $H \rightarrow 0$, from Eq. (10) we can approximate bottom shear stress as $\tau_{b} \approx \tau_{y}$. By substituting Eq. (14) into the governing equations, we can rewrite the governing equations in the case of the limit of low plastic viscosity as follows,

Continuity equation,

$$
\frac{\partial h}{\partial t}+\frac{\tau_{y}}{2 \mu} \frac{\partial H^{2}}{\partial x}=0
$$

Momentum equation,

$$
\frac{\tau_{y}}{2 \mu} \frac{\partial H^{2}}{\partial t}+\left(\frac{\tau_{y}}{2 \mu}\right)^{2} \frac{\partial}{\partial x}\left(\frac{H^{4}}{h}\right)+g \frac{\partial}{\partial x}\left(\frac{h^{2}}{2}\right)=-\frac{\tau_{y}}{\rho}
$$

\section{3) Transformation into moving coordinate system}

We transform the governing equations of Eq. (15) and Eq. (16) into $\xi$ moving coordinate system, where $\xi$ is defined as follows,

$$
\xi=x / l
$$

$l$ is the front position of the flow shown in Fig. 2.

Continuity equation in $\xi$ coordinate system,

$$
\frac{\partial h}{\partial t}-\frac{\xi}{L} \frac{d l}{d t} \frac{\partial h}{\partial \xi}+\frac{\tau_{y}}{2 \mu} \frac{1}{l} \frac{\partial H^{2}}{\partial \xi}=0
$$

Momentum equation in $\xi$ coordinate system,

$$
\begin{gathered}
\frac{\tau_{y}}{2 \mu}\left[\frac{\partial H^{2}}{d t}-\frac{\xi}{l} \frac{d l}{d t} \frac{\partial H^{2}}{\partial \xi}\right]+\left(\frac{\tau_{y}}{2 \mu}\right)^{2} \frac{1}{l} \frac{\partial}{\partial \xi}\left[\frac{H^{4}}{h}\right] \\
+\frac{g}{l} \frac{\partial}{\partial \xi}\left[\frac{h^{2}}{2}\right]=-\frac{\tau_{y}}{\rho}
\end{gathered}
$$

The flow profile is assumed as follows,

$$
h(t, \xi)=\sqrt{h_{20}+h_{21} \xi+h_{22} \xi^{2}}
$$

The reason for choosing such profile in Eq. (20) is related to the flow profile at static which is derived in Subsection 3.2. The boundary condition for $h(t, \xi)$ is as follows,

$$
h_{20}+h_{21}+h_{22}=0
$$

$h_{20}, h_{21}$, and $h_{22}$ are time dependent coefficients. It should be noted that $h_{20}$ is also the depth at the origin. The shear-flow layer $H$ is assumed to have the following profile,

$$
H^{2}=H_{1} \xi+H_{2} \xi^{2}
$$

The boundary condition for $H$ is as follows,

$$
H_{1}+H_{2}=0
$$

$H_{1}$ and $H_{2}$ are time dependent coefficients.

We substitute Eq. (20) and Eq. (22) into the governing equations of Eq. (18) and Eq. (19). We can therefore write the following relations based on the order of $\xi$.

From $\xi^{0}$ order of continuity equation,

$$
\left(\frac{\partial h_{20}}{\partial t}\right)^{2}=\left(\frac{\tau_{y}}{\mu}\right)^{2} \frac{1}{l^{2}} H_{1}^{2} h_{20}
$$

From $\xi^{0}$ order of momentum equation,

$$
h_{21}=-\frac{\tau_{y}}{\rho g} 2 l
$$

From $\xi^{1}$ order of momentum equation,

$$
\frac{\tau_{y}}{2 \mu}\left[\frac{\partial H_{1}}{\partial t}-\frac{H_{1}}{l} \frac{d l}{d t}+\left(\frac{\tau_{y}}{2 \mu}\right) \frac{2 H_{1}^{2}}{l \sqrt{h_{20}}}\right]+\frac{g}{l} h_{22}=0
$$

We substitute $h_{22}=-\left(h_{20}+h_{21}\right)$ from Eq. (22) into Eq. (20). By re-arranging Eq. (20), we have

$$
\begin{aligned}
h & =\sqrt{h_{20}} \sqrt{1-\xi} \sqrt{1+\left(1+\frac{h_{21}}{h_{20}}\right) \xi} \\
& =\sqrt{h_{20}} \sqrt{1-\xi}\left[1+\frac{1}{2}\left(1+\frac{h_{21}}{h_{20}}\right) \xi\right]
\end{aligned}
$$

We consider the volume conservation to obtain a relation between $h_{20}$ and $h_{21}$ as follows, 


$$
v o l=\int_{0}^{l} h d x=\sqrt{h_{20}} l\left(\frac{4}{5}+\frac{2}{15} \frac{h_{21}}{h_{20}}\right)
$$

We use Eq. (24) to substitute $H_{1}$ in Eq. (26) to obtain the following equation,

$$
l \frac{d^{2} \sqrt{h_{20}}}{d t^{2}}+\frac{2 l}{\sqrt{h_{20}}}\left(\frac{d \sqrt{h_{20}}}{d t}\right)^{2}-\frac{g}{l}\left(h_{20}+h_{21}\right)=0
$$

We use the expression of $h_{21}$ in Eq. (25) and substitute it into Eq. (28) and Eq. (29) to obtain the following Eq. (30) and Eq (31) where the only unknowns are $h_{20}$ and $l$.

From Eq. (28)

$$
v o l=\frac{4}{5} \sqrt{h_{20}} l-\frac{4}{15} \frac{\tau_{y}}{\rho g} \frac{l^{2}}{\sqrt{h_{20}}}
$$

From Eq. (29)

$$
\begin{aligned}
& l^{2} \sqrt{h_{20}} \frac{d^{2} \sqrt{h_{20}}}{d t^{2}}+2 l\left(\frac{d \sqrt{h_{20}}}{d t}\right)^{2} \\
& -g h_{20} \sqrt{h_{20}}+\frac{2 \tau_{y}}{\rho} l \sqrt{h_{20}}=0
\end{aligned}
$$

\section{4) Solution of coefficients}

Approximate solutions for Eq. (30) and ODE of Eq. (31) are obtained by the following perturbation method,

We express $\sqrt{h_{20}}$ and $l$ in the following forms,

$$
\begin{gathered}
\sqrt{h_{20}}=H_{0}+a_{1} t \\
l=L_{0}+b_{1} t
\end{gathered}
$$

$H_{0}$ and $L_{0}$ are the initial depth and length shown in Fig. 2. $a_{1}$ and $b_{1}$ are time dependent coefficients.

By substituting Eq. (32) and Eq. (33) into Eq. (30) and OED of Eq. (31), we obtain the following relations based on the order of $t$,

From Eq. (31), $t^{0}$ order,

$$
a_{1}= \pm \sqrt{\frac{g H_{0}^{3}-\frac{2 \tau_{y}}{\rho} H_{o} L_{0}}{2 L_{0}^{2}}}
$$

From Eq. (30), $t^{0}$ order,

$$
H_{0} v o l=\frac{4}{5} H_{0}^{2} L_{0}-\frac{4}{15} \frac{\tau_{y}}{\rho g} L_{0}^{2}
$$

From Eq. (30), $t^{1}$ order,

$$
b_{1}=-\frac{-\frac{3}{5} v o l}{\left(\frac{4}{5} H_{o}^{2}-\frac{8}{15} L_{0} \frac{\tau_{y}}{\rho g}\right)} a_{1}
$$

We therefore solve $a_{1}$ and $b_{1}$ while making sure that Eq. (35) is satisfied as well. Consequently, the time development of front position of the flow can be expressed using Eq. (33).

\subsection{Final length of flow}

Due to the existence of yield stress $\tau_{y}$ in Bingham fluid, the flow will ultimately reach a final length, $l_{f}$ where static condition is achieved. Under the static condition, the following equilibrium governs,

$$
g h \frac{\partial h}{\partial x}=-\frac{\tau_{b}}{\rho}=-\frac{\tau_{y}}{\rho}
$$

Under static condition, from the definition in Eq.(10), $\tau_{b}=\tau_{y}$. We integrate Eq. (37) with boundary condition $x=l_{f}, h=0$ to obtain the following flow profile at static,

$$
h(x)=\sqrt{\frac{2 \tau_{y}}{\rho g}} \sqrt{l_{f}-x}
$$

The flow profile at static in Eq. (38) is similar to the flow profile at static derived for cylindrical coordinate in Kokoda et al.'s work. This flow profile at static is used as a reference to approximate the flow profile in Eq. (20). By substituting $x=0$ in Eq. (38), the final depth at the origin, $h_{f 20}$ can be expressed as follows,

$$
h_{f 20}=\sqrt{\frac{2 \tau_{y} l_{f}}{\rho g}}
$$

We integrate Eq. (38) from $x=0$ to $x=l_{f}$ for total flow volume $v o l$ to obtain the following equation,

$$
v o l=\frac{2}{3} \sqrt{\frac{2 \tau_{y}}{\rho g}} l_{f}^{\frac{3}{2}}
$$

Therefore, we can express the final length of flow $l_{f}$ as follows,

$$
l_{f}=\left(\frac{3}{2} v o l \sqrt{\frac{\rho g}{2 \tau_{y}}}\right)^{\frac{2}{3}}
$$

\subsection{Interpolation of initial stage of flow with final length}

We attempt to bridge the characteristic of inertial phase of flow derived in Section 3.1 with the final length derived in Section 3.2 by expressing $l$ in the following form in Eq. (42). We introduce the symbol $l_{\text {int }}$ as the front position derived here.

$$
l_{\text {int }}=\frac{L_{0}+b_{1} t}{1+b_{2} t}
$$

We assume such form in Eq. (42) based on the fact that as $t \rightarrow \infty, l_{\text {int }} \rightarrow l_{f}$. By using the condition that $\mathrm{t} \rightarrow \infty$, $l_{\text {int }} \rightarrow l_{f}$, we obtain a relation between coefficients $b_{2}$ and $b_{1}$.

$$
b_{2}=b_{1} / l_{f}
$$




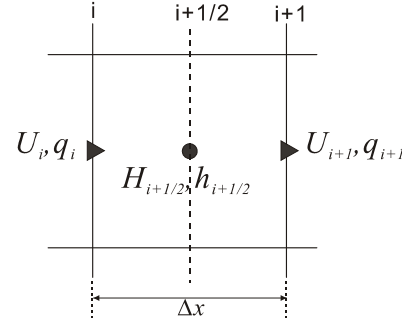

Fig.4 Staggered grid system in the numerical model

Coefficient $b_{1}$ is previously determined in Eq. (36).

\section{Numerical Analysis}

\subsection{Numerical model}

We use a one-dimensional depth-averaged numerical model to verify the theoretical findings by simulating the dam-break flow of finite volume Bingham fluid. The governing equations in the numerical model are the continuity and momentum equations shown in Eq. (11) and Eq. (12).

\section{1) Determining bottom shear stress, $\tau_{b}$}

By rearranging Eq. (13), we obtain a cubic equation as in Eq. (44) where the value of $H$ can be determined.

$$
\frac{\tau_{y}}{2 \mu} H^{3}-3 \frac{\tau_{y}}{2 \mu} h H^{2}+3 U h=0
$$

We determine the bottom shear stress $\tau_{b}$ from Eq. (10). The bottom shear stress $\tau_{b}$ can also be expressed in plug-flow velocity $U_{H}$, and shear-flow thickness $H$ as follows,

$$
\tau_{b}=\tau_{y}+\left.\mu \frac{\partial u}{\partial y}\right|_{y=0}=\tau_{y}+2 \mu \frac{U_{H}}{H}
$$

Therefore, as flow can only be possible under the condition that $\tau_{b}>\tau_{y}$, the suitable value of $H$ determined from Eq. (44) must satisfy the following condition,

$$
H>0
$$

\subsection{Numerical model algorithm}

We use a staggered grid system in the model with the variables defined as in Fig. 4. With $q=U h$ as the discharge per unit width, we define $h^{n+1}, q^{n+1}, U^{n+1}$ and $H^{n+1}$ as the value of $h, q, U$ and $H$ at time step $n+1$. The algorithm in the numerical model is as follows,

First, we determine $h^{n+1}$ from continuity equation in Eq. (11),

$$
h_{i+\frac{1}{2}}^{n+1}=h_{i+\frac{1}{2}}^{n}-\frac{q_{i+1}^{n}-q_{i}^{n}}{\Delta x} \Delta t
$$

Then we evaluate bottom shear stress from momentum equation

$$
\tau_{b}=-\left(\frac{\partial U h}{\partial t}+\frac{\partial U^{2} h}{\partial x}+g h \frac{\partial h}{\partial x}\right)
$$

Table 1. Numerical simulation conditions

\begin{tabular}{|c|c|c|}
\hline \multirow{4}{*}{$\begin{array}{c}\text { Initial } \\
\text { setting }\end{array}$} & $L_{0}(\mathrm{~m})$ & 0.1 \\
\cline { 2 - 3 } & $H_{0}(\mathrm{~m})$ & 0.1 \\
\cline { 2 - 3 } & $\Delta x(\mathrm{~m})$ & 0.005 \\
\cline { 2 - 3 } & $\Delta t(\mathrm{~s})$ & 0.000005 \\
\hline \hline \multirow{2}{*}{$\begin{array}{c}\text { Simulation } \\
\text { cases }\end{array}$} & $\rho\left(\mathrm{kg} / \mathrm{m}^{3}\right)$ & 2187.0 \\
\hline Case $1 a$ & $\tau_{y}(\mathrm{~Pa})$ & $\mu($ Pa.s $)$ \\
\hline Case $1 b$ & 10.0 & 1.0 \\
\hline Case $1 c$ & 10.0 & 10.0 \\
\hline Case $2 a$ & 10.0 & 100.0 \\
\hline Case $2 b$ & 50.0 & 1.0 \\
\hline Case $2 c$ & 50.0 & 10.0 \\
\hline Case 3 & 50.0 & 100.0 \\
\hline Case 4 & 100.0 & 10.0 \\
\hline
\end{tabular}

We use $h^{n+1}, h^{n}$ and $U^{n}$ in the finite difference term of Eq. (48). The value of $\tau_{b}$ evaluated from Eq. (48) is just an approximation since $U^{n}$ is used in the first and second terms on the right hand side of Eq. (48) instead of using $U^{n+1}$. We make sure that the numerical model satisfies the no-flow condition when $\tau_{b}$ evaluated in Eq. (48) is less or equal to the yield stress of the fluid $\tau_{y}$. If $\tau_{b}$ is greater than yield stress $\tau_{y}$, we determine $H^{n+1}$ from Eq. (44) as follows,

$\frac{\tau_{y}}{2 \mu}\left(H^{n+1}\right)^{3}-\frac{3 \tau_{y}}{2 \mu} h^{n+1}\left(H^{n+1}\right)^{2}+3 U^{n} h^{n+1}=0$

By using $H^{n+1}$ from Eq. (49), we use Eq. (45) to determine the bottom shear stress $\tau_{b}$ as follows,

$$
\tau_{b}=\tau_{y} \frac{h_{i+\frac{1}{2}}^{n+1}}{\left(h_{i+\frac{1}{2}}^{n+1}-H^{n+1}\right)}
$$

We determine $q^{n+1}$ from the momentum equation in Eq. (12) as follows,

$$
q_{i}^{n+1}=q_{i}^{n}-\Delta t\left(\text { flux }+ \text { pressure }+\tau_{b}\right)
$$

The flux term is calculated using the first order upwind scheme. The flux term is defined as follows,

$$
\begin{gathered}
f l u x=\frac{U_{i+\frac{1}{2}}^{n} q_{i+a}^{n}-U_{i+\frac{1}{2}}^{n} q_{i-1+b}^{n}}{\Delta x} \\
a=\left\{\begin{array}{l}
0, U_{i+\frac{1}{2}}^{n} \geq 0 \\
1, U_{i+\frac{1}{2}}^{n}<0
\end{array}, \quad b=\left\{\begin{array}{l}
0, U_{i-\frac{1}{2}}^{n} \geq 0 \\
1, U_{i-\frac{1}{2}}^{n}<0
\end{array}\right.\right.
\end{gathered}
$$

The pressure term is defined as follows,

$$
\text { pressure }=g \frac{\left(h_{i+\frac{1}{2}}^{n}+h_{i-\frac{1}{2}}^{n}\right)}{2} \frac{\left(h_{i+\frac{1}{2}}^{n}-h_{i+\frac{1}{2}}^{n}\right)}{\Delta x}
$$

We upgrade $U^{n+1}$ as follows, 


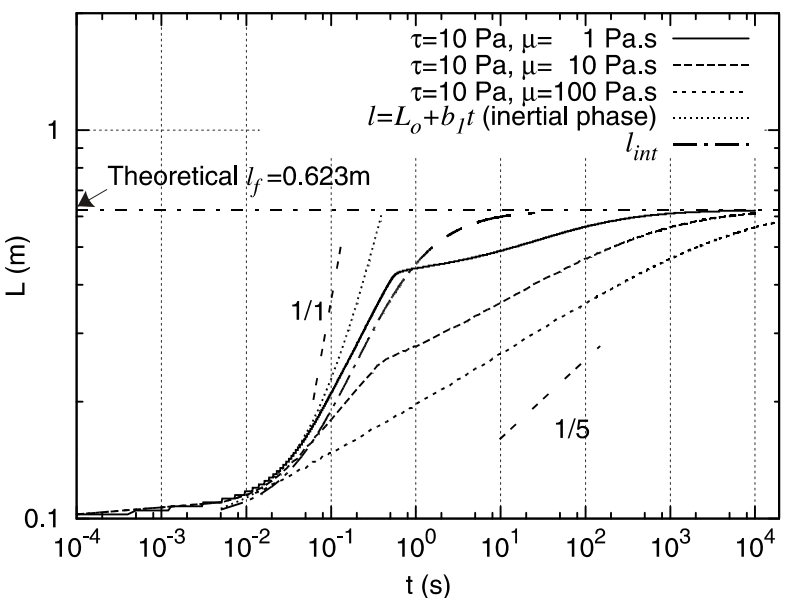

Fig.5 Time development of front position $l$ for Case 1a-1c

$$
U_{i}^{n+1}=q_{i}^{n+1} /\left(h_{i-\frac{1}{2}+c}^{n+1}\right), c=\left\{\begin{array}{l}
0, q_{i}^{n+1} \geq 0 \\
1, q_{i}^{n+1}<0
\end{array}\right.
$$

Numerical simulation conditions are shown in Table 1 where value of plastic viscosity $\mu$ are varied under constant value of yield stress $\tau_{y}$ for Case 1a)-1c) and Case 2a)-2c). In Case 3, a high yield stress value is used, while in Case $4, \tau_{y}=0$ is used to simulate Newtonian fluid. Courant number, $\mathrm{u} \Delta \mathrm{t} / \Delta \mathrm{x}<1$ condition is maintained throughout the simulation.

\section{Results and Discussion}

The simulation results are shown in Fig. 5 and Fig. 6. It can be seen from both figures that the flow reaches a static condition. The final length agrees with the theoretical values in Eq. (41) except for Case 2a) where the final length is $0.389 \mathrm{~m}$, which is an overshoot of $25 \mathrm{~mm}$ from the theoretical value of final length of $0.364 \mathrm{~m}$. At present, we are still trying to improve the numerical model to overcome the overshooting of final length of Case 2a). Both cases in Fig. 5 and Fig. 6 show that with the decrease of plastic viscosity, the characteristic of the inertial phase approaches the asymptotic curve derived from the theoretical analysis in Chapter 3 where $L \propto t$, which obey the $1 / 1$ slope when plotted in logarithmic scale graph. This $1 / 1$ slope is coincidentally same with the characteristic for cylindrical coordinate case $^{2)}$ which is shown in Fig. 1. As the plastic viscosity increases, we could not observe any inertial characteristic. This is due to the fact that more energy is needed to overcome the viscosity of the fluid in the initial stage.

In the author's previous work ${ }^{8)}$, the characteristic of viscous phase for Newtonian fluid is found to be $L \propto t^{1 / 5}$ for problem set in Cartesian coordinate system. As shown in both Fig. 5 and Fig. 6, the viscous phase of Bingham fluid does not agree with the viscous phase of Newtonian fluid. Different value of yield stress produces different characteristic of viscous phase. In order to clarify this, we carried out simulation for Case 3 and Case 4 to study the effect of yield stress $\tau_{y}$ on viscous phase

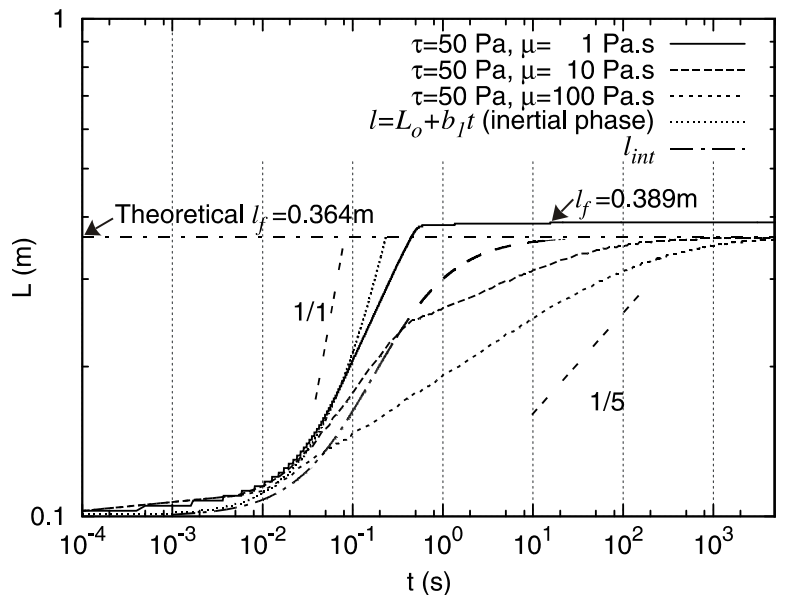

Fig.6 Time development of front position $l$ for Case 2a-2c

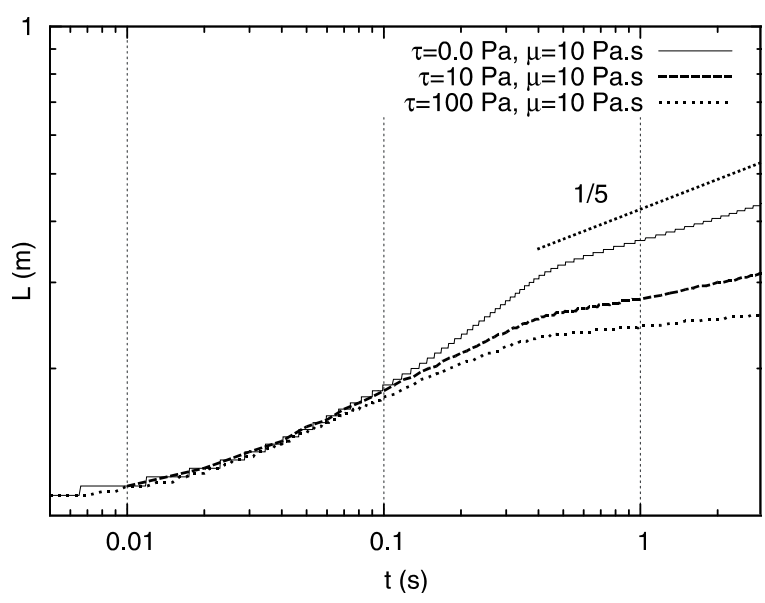

Fig.7 Time development of front position $l$ for Case 3 and 4

characteristic. Fig. 7 shows the comparison of the simulation results. Under constant plastic viscosity, we found that the yield stress $\tau_{y}$ affects the characteristic of the viscous phase. This explains the time development of flow in viscous phase in Fig. 5 and Fig. 6 where the characteristics of viscous phase differ under different yield stress values. In addition, with the decrease of yield stress $\tau_{y}$, the viscous phase characteristic of Bingham fluid approaches the viscous phase characteristic of Newtonian fluid.

\section{Conclusion}

In this paper, we studied the characteristics of inertial phase dam-break flow of low plastic viscosity Bingham fluid theoretically by using simple assumption of plug-flow and shear flow layers in the flow model. In the numerical analysis, we evaluated the bottom shear stress based on the Bingham constitutive relation derived in the theoretical analysis for depth-averaged model. We found satisfactory agreements between the theoretical analysis and numerical results. In the case of low plastic viscosity Bingham fluid, the characteristic of inertial phase approaches an asymptotic solution where $L \propto t$. 
We also found that the characteristic of viscous phase of Bingham fluid is dependent on the value of the yield stress. We plan to extend this work for a wider range plastic viscosity value of Bingham fluid in the future.

\section{References}

1) Krone, R. B., A study of rheologic properties of estuarial sediments, Hydr. Engrg. Lab. and Sanitary Res. Lab., Univ. of Calif., Berkeley, Calif., Ser. Rep. No 63-8, 1963.

2) Hosoda, T., Kokado, T. and Miyagawa, T., Flow characteristics of viscous fluids on the basis of self-similarity law and its applications on high flow concrete, J. App. Mech. JSCE, Vol 3, pp.313-321, 2000. (in Japanese)

3) Kokado, T., Hosoda, T. and Miyagawa, Method of obtaining rheological coefficients of high yield concrete with numerical analysis, Concrete Library of JSCE, Vol. 38, pp.51-70, 2001.
4) Kokado, T., Hosoda, T., Miyagawa, T. and Fujii, M., Study on a method of obtaining yield values of fresh concerete, Concrete Library of JSCE, Vol. 32, pp.29-42, 1998.

5) Huppert, H. E., Flow and instability of a viscous current down a slope, Nature, Vol. 300, pp.427, 1982.

6) Liu, K. F. and Mei, C. C., Slow spreading of a sheet of Bingham fluid on an inclined plane, J. Fluid Mech., Vol. 207,pp.505-529, 1989.

7) Jiang, L. and LeBlond, P. H., Numerical modeling of an underwater Bingham plastic mudslide and the wave which it generates, J. Geophys. Res.,98(C6), pp.10303-10317,1993.

8) Puay, H. T. and Hosoda, T., Study on characteristics of inertia and viscous flow regions by means of dam break flow with finite volume, J. App. Mech. JSCE, Vol 10, pp.757-768, 2007.

(Received: March 8, 2011) 